\title{
Derecho laboral andino
}

\author{
Andean labour law
}

\author{
Direito do trabalho andino
}

\section{Fabián Enrique Vilar Rubiano ${ }^{1}$}

Recibido: 25 de julio de 2021

Aprobado: 17 de octubre de 2021

Publicado: 24 de diciembre de 2021

Cómo citar este artículo:

Fabián Enrique Vilar Rubiano. Derecho laboral andino.

DIXI, vol. 24, $n^{\circ}$. 1, enero-junio 2022, 1-17.

DOI: https://doi.org/10.16925/2357-5891.2022.01.05

Artículo de investigación. https://doi.org/10.16925/2357-5891.2022.01.05

1 Profesor de la Facultad de Derecho de la Universidad Católica de Colombia. Abogado, especialista en Contratación Internacional y magíster en Integración y Globalización de la Universidad Externado de Colombia.

Correo electrónico: fevilar@ucatolica.edu.co 


\section{Resumen}

El Sistema Andino de Integración (SAI) está compuesto por más de diez organismos distribuidos en diferentes países que desarrollan temáticas variadas. En cada una de dichas organizaciones, se ponen en práctica las misiones en pro de la integración. De esta forma, se desarrollan relaciones de trabajo sui generis que, por su dificultad de ejecución, tienen alta opción de terminar en controversias, las cuales deben ser resueltas de manera adecuada para que no afecten la misión de la entidad y la coordinación entre el sistema en general. El presente artículo abordará de manera preliminar la relación entre el derecho laboral internacional y el derecho laboral interno en el marco del SAI para profundizar a través de controversias el análisis de su relación con los principios comunes en el ámbito de la Organización Internacional del Trabajo (OIT).

Palabras clave: derecho laboral andino, integración, Organización Internacional del Trabajo, debido proceso, derechos humanos.

\section{Abstract}

The Andean Integration System (AIS) is composed of more than ten organizations distributed in different countries that develop various topics. In each of these organizations, missions in favor of integration are put into practice. In this way, sui generis working relationships are developed which, due to their difficulty of execution, have a high possibility of ending in controversies, which must be resolved in an adequate manner so that they do not affect the mission of the entity and the coordination between the system in general. This article will preliminarily address the relationship between international labor law and domestic labor law in the framework of the AIS in order to deepen through controversies the analysis of their relationship with the common principles within the scope of the International Labor Organization (ILO).

Keywords: Labor law, Andean, integration, International Labor Organization, due process, human rights.

\section{Resumo}

O Sistema Andino de Integração (SAI) é composto por mais de dez organizações distribuídas em diferentes países que desenvolvem uma variedade de temas. Em cada uma dessas organizações, as missões em favor da integração são colocadas em prática. Desta forma, são desenvolvidas relações de trabalho sui generis que, devido à sua dificuldade de execução, têm grande chance de acabar em controvérsias, que devem ser resolvidas de forma adequada para que não afetem a missão da entidade e a coordenação dentro do sistema em geral. Este artigo abordará preliminarmente a relação entre o direito internacional do trabalho e o direito interno do trabalho no âmbito da SAI, a fim de aprofundar, através de disputas, a análise de sua relação com os princípios comuns no âmbito da Organização Internacional do Trabalho (OIT).

Palavras-chave: Direito do trabalho, andino, integração, Organização Internacional do Trabalho, processo justo, direitos humanos. 


\section{INTRODUCCIÓN}

La Comunidad Andina (CAN) es una organización internacional que cuenta con personería jurídica internacional, compuesta en la actualidad por cuatro Países Miembros: Bolivia, Colombia, Ecuador y Perú. Su misión es muy variada, porque abarca muchos asuntos técnicos que abordan temas de integración económica (finanzas públicas y comercio de bienes y servicios), propiedad intelectual, inversiones tanto andinas como extranjeras, y sanidad humana, animal y vegetal, entre otros, así como asuntos sociales, laborales, de seguridad social, de educación, culturales y de identidad. Para atender la variada necesidad de objetivos a cumplir, a lo largo de sus cincuenta años de existencia, la CAN ha creado diferentes organismos, que hoy en día son quince.

De esta forma, los organismos deben cumplir la misión que dentro del sal les haya sido asignada; por lo tanto, su éxito está condicionado a que en el interior se administren las relaciones laborales en las que concurren trabajadores de diferentes nacionalidades, que según si cumplen actividades misionales o de apoyo, son catalogados como funcionarios internacionales o locales. Lo más importante es que las controversias que se presenten sean resueltas por la autoridad competente, el Tribunal de Justicia de la Comunidad Andina (TJCAN), empleando correctamente la normatividad laboral del organismo para los funcionarios internacionales, el derecho laboral de la sede del organismo para los funcionarios locales, y los principios generales del derecho laboral reconocidos por la oıт y los que sean comunes para ambos.

\section{PRINCIPIOS DEL DERECHO LABORAL RECONOCIDOS POR LA OIT}

La OIT, tiene su origen en 1919 en el Tratado de Versalles con posterioridad a la Segunda Guerra Mundial cuando se buscaba consolidar la paz mundial. Uno de sus principales aspectos es el trato digno a los trabajadores y la organización retoma aspectos ya contemplados en la Asociación Internacional de los Trabajadores de 1901. La OIT se constituyó con nueve miembros: Bélgica, Cuba, Checoslovaquia, Francia, Italia, Japón, Polonia, Reino Unido y Estados Unidos. En 1946, la OIT pasó a ser un organismo del sistema la Organización de las Naciones Unidas (ONU); en la actualidad tiene 187 Estados miembros, entre los que se incluyen los que constituyen la CAN ${ }^{1}$. Lo interesante de la OIT, y lo que la hace un organismo internacional sui generis, es que, en lugar de trabajar a través de sus delegados, su funcionamiento opera

1 Lista de Estados membros de la oІт: https://cutt.ly/gT3QquM 
con tres actores fundamentales: Estados miembros, empleadores y trabajadores. De esta forma, se presenta una aplicación de cooperación para el desarrollo en el ámbito laboral a través de sus políticas y convenios acordados, la cual se retroalimenta permanentemente.

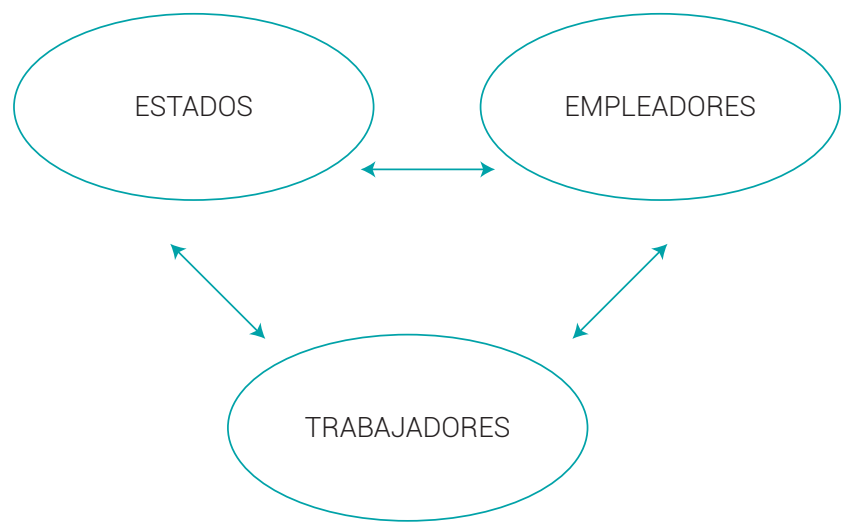

Figura 1. Cooperación tripartita en la OIT

\section{Derecho fundamental de la oIT}

La Conferencia Internacional del Trabajo de la OIT, en su 86. ${ }^{a}$ reunión del 18 de junio de 1998, adoptó la Declaración de la olT Relativa a los Principios y Derechos Fundamentales en el Trabajo y su Seguimiento; la idea es que teniendo en cuenta la globalización acelerada en la economía a partir la década de los noventa, se busca que el desarrollo económico incluya mejoras en la calidad de vida de los trabajadores y su familia ${ }^{2}$. La Declaración contiene cuatro temas que son objeto de protección fundamental:

\section{Libertad de asociación, sindical y negociación colectiva}

Tanto los empleados como los empresarios pueden conformar, sin obstáculos de ninguna índole, agrupaciones que promuevan la cooperación para la protección de los intereses comunes a sus afiliados y administrarlos de manera autónoma en el interior del respectivo grupo, respetando el ordenamiento jurídico que le sea aplicable.

2 Organización Internacional del Trabajo (OIT). Declaración de la oIT relativa a los principios y derechos fundamentales en el trabajo y su seguimiento. 18 de junio de 1998. Disponible en: https://cutt.ly/XT9rVIN 
La organización respectiva puede a su vez afiliarse a grupos de organizaciones afines nacionales e internacionales.

\section{Eliminación de trabajo forzoso}

Es la situación que se presenta cuando el trabajador se ve obligado a trabajar contra su voluntad, ya sea porque se ve compelido por una circunstancia (amenaza, fuerza física, violencia, trabajo con personal ilegal, retención de documentos de identificación, trata de personas, impedimento de libre circulación), porque la paga es exclusivamente en especie o porque le retienen el pago de su remuneración.

\section{Abolición del trabajo infantil}

Los niños son seres humanos, por lo que en principio se les aplica la misma protección que a toda persona; no obstante, es una población que todavía está en etapa de desarrollo en todos los ámbitos (físico, moral, experiencia, etc.) y por eso requiere especial protección con normas más favorables que eviten los riesgos propios de su condición en un entorno laboral. De esta manera, se restringe la utilización de niños para el trabajo en aras de priorizar su desarrollo educativo con escolaridad obligatoria.

\section{Eliminación de discriminación en empleo}

La discriminación es la más común de las manifestaciones que son objeto de protección fundamental y se presenta por cualquier circunstancia en la que se quiera menospreciar a un trabajador y que también puede constituir acoso laboral. La discriminación se presenta por motivos de género, raza, nacionalidad, condición social, religión, costumbres, ideas políticas, condición de discapacidad, enfermedad o cualquier otra situación que permita excluir o perjudicar a una persona en el trabajo.

En consecuencia, la olT tiene con la Declaración un instrumento internacional que desarrolla valores democráticos comunes a los Estados en el ámbito del trabajo, con unas reglas básicas de mínimo común denominador que todos los miembros de la olT deben observar sin excepción y con independencia del número de convenios fundamentales que hayan ratificado.

Todo miembro de la OIT está vinculado por lo menos a la Constitución de la organización y a la Declaración; y además de constituir unas garantías básicas para el trabajador, se genera para los Estados un mecanismo de presión para observar 
los principios relativos a derechos fundamentales que son objeto en los convenios (1930, 1948, 1949, 1951, 1957, 1958, 1973 y 1999)3. Es decir, incluso si el Estado no ha ratificado convenios, de manera indirecta está obligado por los principios en ellos contenidos, lo que a la larga incidirá en la futura ratificación que se haga respecto a convenios fundamentales de la olT.

Tabla 1. Convenios fundamentales de la OIT

\begin{tabular}{|c|c|c|c|c|}
\hline Tema & Número & Convenio & Adopción & En vigor \\
\hline \multirow{2}{*}{$\begin{array}{l}\text { Libertad de asociación, } \\
\text { sindical y negociación } \\
\text { colectiva }\end{array}$} & 87 & $\begin{array}{l}\text { Convenio sobre la libertad sindical y la } \\
\text { protección del derecho de sindicación }\end{array}$ & 9/7/1948 & $4 / 7 / 1950$ \\
\hline & 98 & $\begin{array}{l}\text { Convenio sobre el derecho de sindica- } \\
\text { ción y de negociación colectiva }\end{array}$ & $1 / 7 / 1949$ & $18 / 7 / 1951$ \\
\hline \multirow{2}{*}{$\begin{array}{l}\text { Eliminación de trabajo } \\
\text { forzoso }\end{array}$} & 29 & Convenio sobre el trabajo forzoso & 28/6/1930 & $1 / 5 / 1932$ \\
\hline & 105 & $\begin{array}{l}\text { Convenio sobre la abolición del } \\
\text { trabajo forzoso }\end{array}$ & 25/6/1957 & $17 / 1 / 1959$ \\
\hline \multirow{2}{*}{$\begin{array}{l}\text { Abolición trabajo } \\
\text { infantil }\end{array}$} & 138 & Convenio sobre la edad mínima & 26/6/1973 & $19 / 6 / 1976$ \\
\hline & 182 & $\begin{array}{l}\text { Convenio sobre las peores formas de } \\
\text { trabajo infantil }\end{array}$ & 17/6/1999 & $19 / 11 / 2000$ \\
\hline \multirow{2}{*}{$\begin{array}{l}\text { Eliminación de discrimi- } \\
\text { nación en empleo }\end{array}$} & 100 & $\begin{array}{l}\text { Convenio sobre igualdad de remu- } \\
\text { neración }\end{array}$ & 29/6/1951 & 23/5/1953 \\
\hline & 111 & $\begin{array}{l}\text { Convenio relativo a la discriminación } \\
\text { en materia de empleo y ocupación }\end{array}$ & $25 / 6 / 1958$ & $15 / 6 / 1960$ \\
\hline
\end{tabular}

Fuente: Elaboración propia.

Así mismo, como mecanismo de presión para quienes no hayan ratificado los convenios fundamentales, estarán obligados a presentar a la OIT informes anuales sobre cómo se están aplicando los principios fundamentales de la Declaración. Por su parte, la organización elaborará un informe cuatrianual con los progresos para determinar la eficacia del mecanismo con las acciones ejecutadas y planear las políticas del próximo cuatrienio al respecto.

En el Tercer Considerando de la Declaración', se establece que la OIT se enfocará en la cooperación en asuntos de empleo, lo que consagra su misión funda-

3 Convenio sobre el trabajo forzoso, 28 de junio de 1930.

Convenio sobre la libertad sindical y la protección del derecho de sindicación, 1948.

Convenio sobre el derecho de sindicación y de negociación colectiva, 1949.

Convenio sobre igualdad de remuneración, 1951.

Convenio sobre la abolición del trabajo forzoso, 1957.

Convenio relativo a la discriminación en materia de empleo y ocupación, 1958.

Convenio sobre la edad mínima, 1973.

Convenio sobre las peores formas de trabajo infantil, 1999.

4

OIT, supra, nota 3. 
mental. El Cuarto Considerando de la Declaración remite la atención a personas con necesidades especiales, entre ellas los desempleados y los trabajadores migrantes; entre estos últimos se encuentra la mayoría de los funcionarios internacionales de los organismos del SAI.

\section{Principios fundamentales en los países andinos}

Como muestra de su compromiso por los principios del derecho laboral, todos los Países Miembros de la CAN han ratificado todos los Convenios Fundamentales de la OIT, como se expone en la tabla 2.

Tabla 2. Convenios fundamentales de la OIT en los miembros de la CAN

\begin{tabular}{|c|c|c|c|}
\hline Tema & Número & Convenio & Ratificación \\
\hline \multirow{8}{*}{$\begin{array}{l}\text { Libertad de asociación, sindi- } \\
\text { cal y negociación colectiva }\end{array}$} & \multirow{4}{*}{87} & \multirow{4}{*}{$\begin{array}{l}\text { Convenio sobre la libertad sindical } \\
\text { y la protección del derecho de } \\
\text { sindicación }\end{array}$} & 4/1/1965 Bolivia \\
\hline & & & 16/11/1976 Colombia \\
\hline & & & 29/5/1967 Ecuador \\
\hline & & & 2/3/1960 Perú \\
\hline & \multirow{4}{*}{98} & \multirow{4}{*}{$\begin{array}{l}\text { Convenio sobre el derecho de } \\
\text { sindicación y de negociación } \\
\text { colectiva }\end{array}$} & 15/11/1973 Bolivia \\
\hline & & & 16/11/1976 Colombia \\
\hline & & & 28/5/1959 Ecuador \\
\hline & & & 13/3/1964 Perú \\
\hline \multirow{8}{*}{$\begin{array}{l}\text { Eliminación de trabajo } \\
\text { forzoso }\end{array}$} & \multirow{4}{*}{29} & \multirow{4}{*}{ Convenio sobre el trabajo forzoso } & 31/5/2005 Bolivia \\
\hline & & & 4/3/1969 Colombia \\
\hline & & & 6/7/1954 Ecuador \\
\hline & & & 1/2/1960 Perú \\
\hline & \multirow{4}{*}{105} & \multirow{4}{*}{$\begin{array}{l}\text { Convenio sobre la abolición del } \\
\text { trabajo forzoso }\end{array}$} & 11/6/1990 Bolivia \\
\hline & & & 7/6/1963 Colombia \\
\hline & & & 5/2/1962 Ecuador \\
\hline & & & 6/12/1960 Perú \\
\hline \multirow{8}{*}{ Abolición trabajo infantil } & \multirow{4}{*}{138} & \multirow{4}{*}{ Convenio sobre la edad mínima } & 11/6/1997 Bolivia \\
\hline & & & 2/2/2001 Colombia \\
\hline & & & 19/9/2000 Ecuador \\
\hline & & & 13/11/2002 Perú \\
\hline & \multirow{4}{*}{182} & \multirow{4}{*}{$\begin{array}{l}\text { Convenio sobre las peores formas } \\
\text { de trabajo infantil }\end{array}$} & 6/6/2003 Bolivia \\
\hline & & & 28/1/2005 Colombia \\
\hline & & & 19/9/2000 Ecuador \\
\hline & & & 10/1/2002 Perú \\
\hline
\end{tabular}

(continúa) 


\begin{tabular}{|c|c|c|c|}
\hline Tema & Número & Convenio & Ratificación \\
\hline \multirow{8}{*}{$\begin{array}{l}\text { Eliminación de discriminación } \\
\text { en empleo }\end{array}$} & \multirow{4}{*}{100} & \multirow{4}{*}{$\begin{array}{l}\text { Convenio sobre igualdad de } \\
\text { remuneración }\end{array}$} & 15/11/1973 Bolivia \\
\hline & & & 7/6/1963 Colombia \\
\hline & & & 1/3/1957 Ecuador \\
\hline & & & 1/2/1960 Perú \\
\hline & \multirow{4}{*}{111} & \multirow{4}{*}{$\begin{array}{l}\text { Convenio relativo a la discrimi- } \\
\text { nación en materia de empleo y } \\
\text { ocupación }\end{array}$} & 31/1/1977 Bolivia \\
\hline & & & 4/3/1969 Colombia \\
\hline & & & 10/7/1962 Ecuador \\
\hline & & & 10/8/1970 Perú \\
\hline
\end{tabular}

Fuente: Elaboración propia.

\section{DERECHO LABORAL EN OTROS SISTEMAS DE INTEGRACIÓN}

Es diferente la vinculación laboral en los organismos de otros sistemas de integración, entendidos como los que tienen institucionalidad, nómina propia y la potencialidad de buscar el bienestar de la integración e independencia en sus decisiones frente a los Estados miembros.

El reclutamiento de estos organismos en otros sistemas de integración tiene plena aplicación. De esta manera, el SAI podría implementar las experiencias que en dicho tema le aportan y crear un sistema de carrera administrativa para que el sAI se beneficie atrayendo y reteniendo los mejores funcionarios, beneficiando a la entidad y excluyendo los riesgos de sesgo político, de nacionalidad y nepotismo, entre otros. Los ciudadanos andinos que tengan el deseo y las competencias requeridas pueden acceder a laborar en cualquiera de los organismos del SAI con empleos de calidad y estabilidad.

\section{Unión Europea (UE)}

La UE es el sistema de integración más profundo que existe el mundo. La Secretaría General de la Comunidad Andina (SGCAN) sirve de modelo para lo demás y es particular inspiración para la CAN para la vinculación de los funcionarios de todos y cada uno de sus organismos ${ }^{5}$. El proceso de selección de personal mediante el sistema de concurso de mérito permite una vinculación de carácter permanente; dicho proceso es centralizado y administrado por la Oficina Europea de Selección de Personal (EPSO,

5 Perfiles de instituciones y órganos de la Unión Europea: https://cutt.ly/7T3QxZb 
por sus siglas en inglés), que tiene la misión fundamental de escoger los mejores funcionarios para los organismos de la UE acorde con el Estatuto de funcionarios de la UE.

El proceso de selección consiste, en primer lugar, en unas pruebas que el candidato presenta de manera individual frente a un computador; en caso de ser aprobadas, tendrá otra ronda de examen en grupo sobre aspectos relacionados con el empleo ante un jurado; finalmente, quienes superen las dos etapas integrarán un listado de reserva y serán entrevistados los aspirantes con mejores resultados. Dicha lista la usará el organismo en función de sus necesidades de vinculación, cuya vigencia será de un año con posibilidad de prorrogarse.

Por último, en cuanto a las controversias en materia laboral que se presenten entre la UE y sus funcionarios, el Tribunal de Justicia de la Unión Europea (TJUE) será competente previa reclamación ante el organismo empleador con pretensión rechazada de manera explícita o implícita.

\section{Organización Mundial del Comercio (омC)}

La OMC, que en la actualidad tiene 164 miembros, incluidos todos los Países Miembros de la $\mathrm{CAN}^{6}$, es el foro multilateral donde se concurre para negociar la normatividad en materia de liberalización de comercio exterior y solucionar las diferencias a que haya lugar. En materia laboral, la OMc tiene un sistema de carrera con vinculación indefinida a partir del quinto año de prestación del servicio, con posibilidad de traslados a otras dependencias de la organización.

\section{Mercado Común del Sur (Mercosur)}

El Mercosur, sistema de integración del Cono Sur de América, está constituido por Argentina, Brasil, Paraguay y Uruguay, y tiene como aparato institucional el Fondo para la Convergencia Estructural del Mercosur (Focem), el Instituto de Políticas Públicas en Derechos Humanos (IPPDH), el Instituto Social del Mercosur (ISM), el Parlamento del Mercosur (Parlasur), la Secretaría del Mercosur y el Tribunal Permanente de Revisión (TPR).

En materia laboral, en los organismos del Mercosur la vinculación será en periodo de prueba por un año, con renovaciones de tres años al cabo de la tercera renovación; es decir, diez años en total. Se vincula al funcionario con renovaciones automáticas y las controversias que se presenten al respecto serán resueltas por el Tribunal Administrativo Laboral (TAL).

6 Miembros y observadores de la oMc: https://cutt.ly/dT3QEJM 


\section{DERECHO LABORAL ANDINO}

De los quince organismos que tiene el SAI (figura 2), siete tienen nómina propia compuesta por funcionarios que no dependen de los miembros y desarrollan las actividades permanentes de la respectiva entidad. La SGCAN tiene función ejecutiva, el TJCAN tiene función judicial, el Parlamento Andino tiene función democrática, el Banco de Desarrollo de América Latina (CAF) y el Fondo Latinoamericano de Reservas tienen funciones financieras, la Universidad Andina Simón Bolívar (UASB) tiene función educativa y el Convenio Hipólito Unanue es el organismo andino de salud.

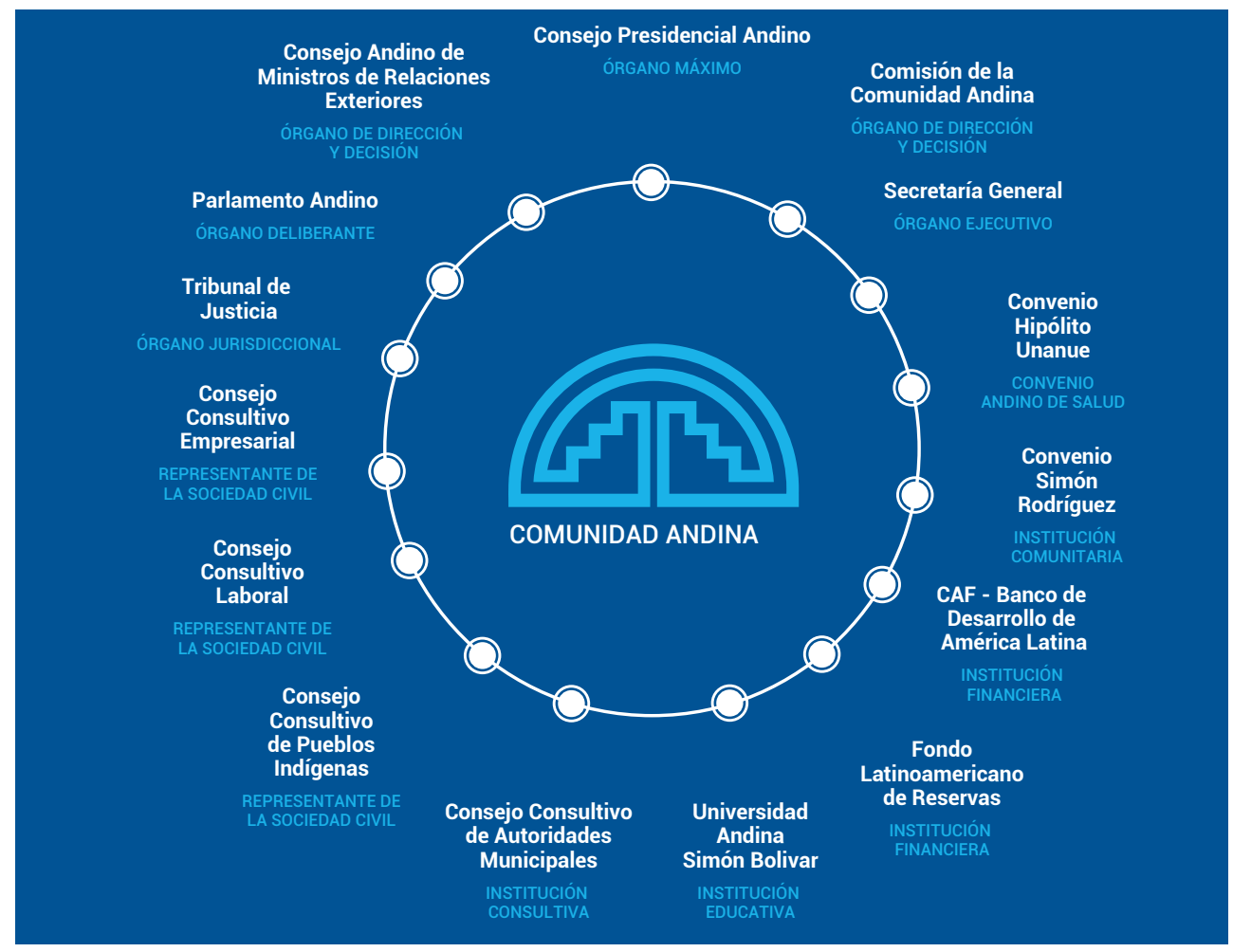

Figura 2. Organismos del SAI

Fuente: Página web de la Comunidad Andina.

Como se desprende de los importantes y variados temas que manejan los organismos, requieren independencia para cumplir con sus funciones. Por esta razón, se necesita que la misión de la entidad sea cumplida por los mejores funcionarios que actúen en beneficio de la integración y con autonomía frente a los intereses de los miembros, incluido por supuesto el Estado de origen del funcionario. 
En todos los organismos del SAI, en materia laboral previa reclamación administrativa ante el empleador con la respuesta denegatoria, denegatoria parcial o la acreditación de no haber obtenido respuesta en un término de treinta días ${ }^{7}$, el TJCAN es la autoridad competente para decidir las controversias que se presenten entre sus funcionarios o exfuncionarios y la respectiva entidad de conformidad con el convenio sede del Estado que la acoge. En la sentencia, el TJCAN utilizará los principios generales del derecho de la oIt y aquellos que sean comunes a los Países Miembros. Durante treinta y siete años (1984 a 2021) solo se han recibido veintidos casos; el número tan reducido obedece a que tiene barreras de acceso muy altas y tiene en cuenta los costos de litigar ante el TJCAN para quienes sean residentes o nacionales de país diferente de la sede del organismo, además de contar con los servicios de abogados expertos en derecho andino. De las controversias descritas, en siete no se conoce al demandado pues no cuentan con publicación en la Gaceta Oficial del Acuerdo de Cartagena (GOAC); en cinco (todas del 2013) porque fueron archivadas al llegar las partes y llegaron a acuerdo conciliatorio (TJCAN); y dos que llegaron a sentencia no se publicaron $^{8}$. Y las quince restantes tienen como demandados a la SGCAN seis veces (TJCAN 75-DL-2003, 13-DL-2005, 81-DL-2005, 1-DL-2008, 3-DL-2018 y 1-DL-2021), a la UASB en cuatro ocasiones (TJCAN 1-DL-2013, 3-DL-2013, 1-DL-2017 y 1-DL-2018), al Parlamento Andino en dos oportunidades (TJCAN 1-DL-2012 y 1-DL-2020); y en un proceso para cada uno el Convenio Hipólito Unanue (TJCAN 1-DL-2009), trabajadores de la SGCAN (TJCAN 73-DL-2003) y la Asociación de Empresas Estatales de Telecomunicaciones del Acuerdo Subregional Andino (TJCAN 2-DL-2017).

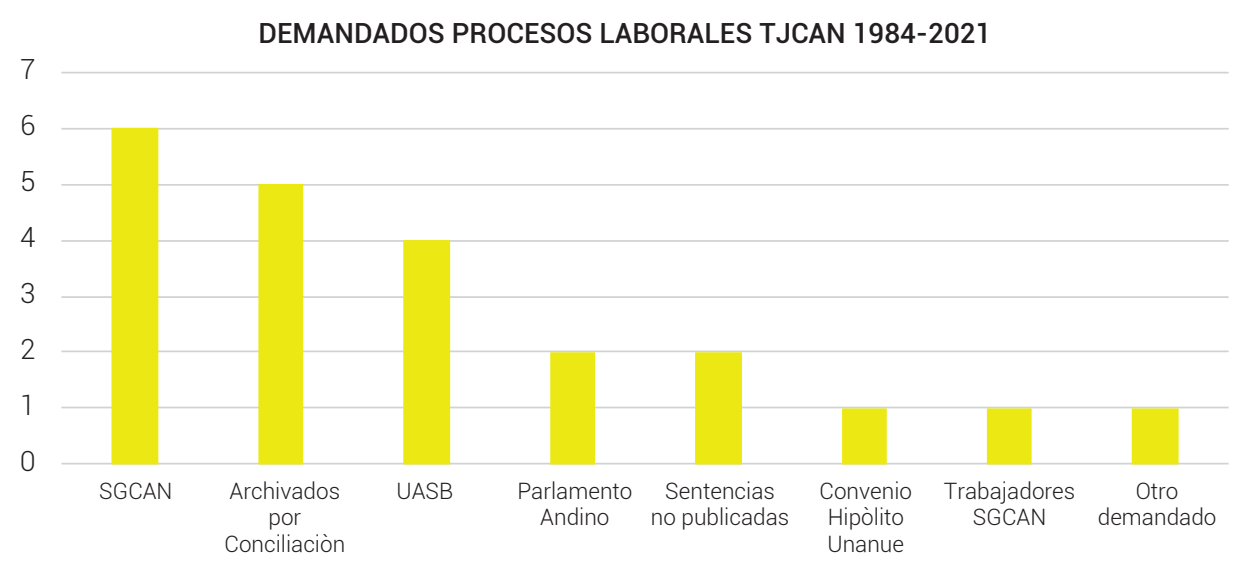

Figura 3. Demandados procesos laborales TJCAN 1984 - 2021 Fuente: Elaboración propia.

7 Comunidad Andina (CAN). manual de procedimientos del sistema andino de SOLUCIÓN DE CONTROVERSIAS. CAN. (2008). Disponible en: https://cutt.ly/zTwMwuH

8 1-DL-2014 y 2-DL-2018. 
En el caso TJCAN 73-DL-2003, la SGCAN activa el proceso y le solicita al TJCAN que la autorice para retener dineros a unos trabajadores. El inicio de la acción demuestra un desconocimiento evidente del demandante del ordenamiento jurídico porque pretendía invertir los papeles; es decir, cada une las partes de la controversia es calificada, pues solo los trabajadores o extrabajadores tienen legitimación activa y solo los organismos del SAI pueden ser los demandados.

Los trabajadores que tienen los organismos del SAI pueden ser de dos clases: funcionarios locales y funcionarios internacionales. La clasificación de los empleados corresponde a la normatividad del organismo (reglamento interno o de funcionarios). Los locales prestan servicio en actividades de apoyo, en aspectos administrativos; los funcionarios internacionales prestan servicio según sean clasificados en la normatividad del organismo, que por regla general son los de nivel directivo; y los funcionarios del nivel técnico-científico desarrollan actividades misionales de la entidad.

En consecuencia, por la variada naturaleza de las funciones, a cada categoría de funcionarios se le aplica normatividad laboral diferente. Los empleados locales son sujetos del derecho laboral del país sede del organismo y a los funcionarios internacionales se les implementa la normatividad que al respecto haya establecido la entidad, en particular, pero no exclusivamente, su reglamento interno y contrato.

El juez andino en sentencia del proceso 1DL-201 ha reconocido en su jurisprudencia el Convenio oIT N ${ }^{\circ} 182$ sobre la terminación de la relación de trabajo, del cual se desprenden principios generales del derecho laboral como justa causa de despido (artículo 4) y derecho de defensa (artículo 7). Al respecto, el TJCAN ha establecido: "Así, pues, el Convenio sobre la terminación de la relación de trabajo de la Organización Internacional del Trabajo (OIT) de 1982 señala al efecto lo siguiente: 'No se pondrá término a la relación de trabajo de un trabajador a menos que exista para ello una causa justificada relacionada con su capacidad o su conducta o basada en las necesidades de funcionamiento de la empresa, establecimiento o servicio'" (artículo 4).

Por su parte, el artículo 7 del mismo cuerpo normativo señala que: "No deberá darse por terminada la relación de trabajo de un trabajador por motivos relacionados con su conducta o su rendimiento antes de que se le haya ofrecido la posibilidad de defenderse de los cargos formulados contra él, a menos que no pueda pedirse razonablemente al empleador que le conceda esta posibilidad"9.

De tal manera, en caso de contemplar la posibilidad de despedir funcionarios, previo a su desvinculación, los organismos del SAI deben observar lo dispuesto por la normatividad de la oIT respecto a la terminación de la relación de trabajo y acogido

9 Tribunal de Justicia de la Comunidad Andina (TJCAN). (2013). Gestión 2013. Disponible en: https://cutt.ly/kT9tgMT 
expresamente por el TJCAN, que tendrá en cuenta dicha jurisprudencia precedente para los procesos que lleguen a su conocimiento.

Acerca del cumplimiento de los principios descritos en la Resolución 701, la Resolución 744, la Resolución 745, la Resolución 746 y la Resolución 747, la entidad empleadora adelanta una investigación respetando las debidas garantías para concluir en la destitución de los funcionarios por faltas graves ${ }^{10}$. Sin embargo, la SGCAN no siempre cumple el derecho laboral andino para despedir funcionarios. Para la muestra, en el proceso 3-DL-2018 (actualmente en la última etapa faltando sólo la sentencia) la SGCAN fue demandada con dos tesis:

- La primera es que el despido fue injusto porque al demandante no se le notificó calificación de desempeño negativa, no le endilgaron falta alguna, mucho menos grave, y no tuvo oportunidad de defenderse o presentar descargos frente al despido ${ }^{11}$.

- La segunda es que la SGCAN tiene obligaciones internacionales, entre ellas respetar los principios generales del derecho laboral reconocidos por la OIT y los derechos humanos, en particular las garantías judiciales en materia laboral que son aplicables a toda instancia procesal, verbigracia de naturaleza administrativa para contestar el pliego de cargos, que en este caso no hubo. Dicho caso, cuando sea resuelto (lo más probable en 2022), será una jurisprudencia que sentará un precedente en el derecho laboral andino por parte del TJCAN.

\section{PRopuesta de MEjora}

Finalmente, aprendiendo de las buenas prácticas de otros mecanismos de administración laboral en diferentes sistemas de integración y rescatando el valioso acervo jurídico e institucional que en materia laboral ya tiene el SAI, se propone que, para atraer y retener el mejor talento posible, la CAN implemente un sistema de carrera de carácter permanente tal como existe en la UE y la OMC, con vinculación a los

10 Resolución 701 (28 de febrero de 2003), Resolución 744 (23 de julio de 2003), Resolución 745 (23 de julio de 2003), Resolución 746 (23 de julio de 2003) y Resolución 747 (23 de julio de 2003).

11 Durante todo el proceso que lleva tres años (2018 a 2021), la SGCAN no presentó pruebas de la evaluación de desempeño insatisfactoria, ni llamados de atención al trabajador ni acreditó haber realizado previo al despido trámite administrativo disciplinario con observancia del principio universal del derecho de garantía al debido proceso en ninguna de sus actuaciones procesales: contestación de la demanda, audiencia de conciliación, audiencia de alegatos de conclusión y escrito de conclusiones. 
organismos del SAI por estricto sistema de mérito y respetando la distribución geográfica equitativa de procedencia de los funcionarios. Debe tener posibilidad de traslado tanto en el interior de la entidad como a otra institución andina con un sistema centralizado de selección y promoción de carrera para todo el SAI, a través de la supervisión de una entidad nueva que se cree para ello y que se incorpore al sistema o con el fortalecimiento de la Oficina de Recursos Humanos de la SGCAN. En cualquiera de las opciones, se debe vincular en el proceso de selección a la entidad y dependencia receptora de los aspirantes.

\section{CONCLUSIONES}

En controversias laborales en organismos del SAl, el juez competente es el TJCAN y las fuentes aplicables son las normas laborales del organismo para los funcionarios internacionales, el derecho laboral del país sede lo es para los funcionarios locales, así como los principios generales del derecho laboral reconocidos por la oIT y los principios generales de derecho laboral comunes a los Países Miembros de la CAN para ambos.

El derecho laboral andino es una muestra de relación, integración, cooperación y valores democráticos comunes entre la normatividad sobre relaciones de trabajo de carácter local, andino e internacional. El ordenamiento jurídico laboral andino sigue los principios generales de la OIT, tales como el despido justificado en causal probada y el derecho de defensa.

Se debe tener especial consideración con el trabajador migrante andino de los organismos del SAI, dadas sus especiales circunstancias pues al salir de su zona de confort debe enfrentarse a situaciones desconocidas como otra cultura y costumbres tanto del lugar de destino como de sus compañeros y jefes. Para que se preste el mejor servicio posible, es necesario atraer y especialmente retener a los mejores funcionarios cuya formación, experiencia y competencias favorezcan la misión del organismo del SAI.

Con el propósito de evitar duplicidad y tener claridad en las competencias y lo que se debe y no se debe hacer en la actividad laboral, todos y cada uno de los organismos del SAI deben contar con un manual de funciones detalladas para la entidad, las direcciones u oficinas y para cada uno de los funcionarios, así como actualizarlo permanentemente.

En aras de conseguir la mejor calidad en el servicio por parte de la entidad y estabilidad en el empleo del trabajador, sería adecuado que los funcionarios de los 
organismos del SAI tengan un sistema de carrera de carácter permanente con movilidad y promoción en el interior de la entidad y con otro organismo del sistema.

El TJCAN tiene la misión fundamental de aplicar correctamente el derecho laboral andino y su jurisprudencia, mediante la impartición de una pronta y debida justicia en las demandas laborales que se sometan a su consideración contra cualquiera de los organismos del SAI.

\section{REFERENCIAS}

Comunidad Andina (can). Manual de Procedimientos del Sistema Andino de Solución de CONTROVERSIAS. CAN. (2008). Disponible en: https://cutt.ly/zTwMwuH

Organización Internacional del Trabajo (OIT). Convenio sobre el trabajo forzoso. 28 de junio de 1930. Disponible en: https://cutt.ly/HT9rp1x

Organización Internacional del Trabajo (OIT). Convenio sobre la libertad sindicaly la protección del derecho de sindicación. 1948. Disponible en: https://cutt.ly/kT9rlJb

Organización Internacional del Trabajo (OIT). Convenio sobre el derecho de sindicación y de negociación colectiva. 1949. Disponible en: https://cutt.ly/dT9rid0

Organización Internacional del Trabajo (ОІт). Convenio sobre igualdad de remuneración. 1951. Disponible en: https://cutt.ly/PT9rsDI

Organización Internacional del Trabajo (OIT). Convenio sobre la abolición del trabajo forzoso. 1957. Disponible en: https://cutt.ly/fT9rhqT

Organización Internacional del Trabajo (OIT). Convenio relativo a la discriminación en materia de empleo y ocupación. 1958. Disponible en: https://cutt.ly/8T9ryFd

Organización Internacional del Trabajo (OIT). Convenio sobre la edad mínima. 1973. Disponible en: https://cutt.ly/nT9rjv2

Organización Internacional del Trabajo (ОІт). Convenio sobre las peores formas de trabajo infantil. 1999. Disponible en: Disponible en: https://cutt.ly/YT9rxyH

Organización Internacional del Trabajo (OІT). Declaración de la oIT relativa a los principios y derechos fundamentales en el trabajo y su seguimiento. 18 de junio de 1998. Disponible en: https:// cutt.ly/XT9rVIN 
Secretaría General de la Comunidad Andina (SGCAN). Resolución 701 de 2003. (28 de febrero de 2003). Disponible en: https://cutt.ly/JT9teCl

Secretaría General de la Comunidad Andina (SGCAN). Resolución 744 de 2003. (23 de julio de 2003). Disponible en: https://cutt.ly/hT9ttdP

Secretaría General de la Comunidad Andina (SGCAN). Resolución 745 de 2003. (23 de julio de 2003). Disponible en: https://cutt.ly/YT9twsb

Secretaría General de la Comunidad Andina (SGCAN). Resolución 746 de 2003. (23 de julio de 2003). Disponible en: https://cutt.ly/3T9tqyz

Secretaría General de la Comunidad Andina (SGCAN). Resolución 747 de 2003. (23 de julio de 2003). Disponible en: https://cutt.ly/zT9r6ez

Tribunal de Justicia de la Comunidad Andina (TJCAN). Auto 73-DL-2003. (19 de agosto de 2003). Disponible en: https://cutt.ly/VT9r5jq

Tribunal de Justicia de la Comunidad Andina (TJCAN). Sentencia 75-DL-2003. (19 de agosto de 2003). Disponible en: https://cutt.ly/xT9r7sa

Tribunal de Justicia de la Comunidad Andina (TJCAN). Auto 13-DL-2005. (13 de septiembre de 2006). Disponible en: https://cutt.ly/TT9tpAw

Tribunal de Justicia de la Comunidad Andina (TJCAN). Sentencia 81-DL-2005. (5 de diciembre de 2006). Disponible en: https://cutt.ly/9T9tosG

Tribunal de Justicia de la Comunidad Andina (TJCAN). Sentencia 1-DL-2008. (4 de marzo de 2010). Disponible en: https://cutt.ly/IT9ts9L

Tribunal de Justicia de la Comunidad Andina (TJCAN). Sentencia 1-DL-2009. (18 de abril de 2012). Disponible en: https://cutt.ly/ST9tf00

Tribunal de Justicia de la Comunidad Andina (TJCAN). (2013). Gestión 2013. Disponible en: https:// cutt.ly/kT9tgMT

Tribunal de Justicia de la Comunidad Andina (TJCAN). Sentencia 1-DL-2012. (17 de abril de 2013). Disponible en: https://cutt.ly/jT9th5A

Tribunal de Justicia de la Comunidad Andina (TJCAN). Sentencia 1-DL-2013. (28 de noviembre de 2014). Disponible en: https://cutt.ly/UT9tkC2 
Tribunal de Justicia de la Comunidad Andina (TJCAN). Sentencia 3-DL-2013. (21 de enero de 2015). Disponible en: https://cutt.ly/WT9tzfP

Tribunal de Justicia de la Comunidad Andina (TJCAN). Auto 1-DL-2017. (3 de octubre de 2017). Disponible en: https://cutt.ly/aT9txVQ

Tribunal de Justicia de la Comunidad Andina (TJCAN). Auto 2-DL-2017. (11 de mayo de 2018). Disponible en: https://cutt.ly/dT9r8P9

Tribunal de Justicia de la Comunidad Andina (TJCAN). Sentencia 1-DL-2018. (8 de abril de 2019). Disponible en: https://cutt.ly/XT9r9D2

Tribunal de Justicia de la Comunidad Andina (TJCAN). Auto 3-DL-2018. (29 de mayo de 2020). Disponible en: https://cutt.ly/wT9r2wU

Tribunal de Justicia de la Comunidad Andina (TJCAN). Auto 1-DL-2020. (19 de noviembre de 2012). Disponible en: https://cutt.ly/WT9r1dU 